\title{
Spatial-temporal HEVC Inter-prediction Error Concealment
}

\author{
Xiangqun li ${ }^{1,2, a}$, Xiaohai $\mathrm{He}^{1, \mathrm{~b}}$, Wenhui Jing ${ }^{3, \mathrm{c}}$, Xin Peng ${ }^{1, \mathrm{~d}}$ \\ ${ }^{1}$ College of Electronics and Information Engineering, Sichuan University, Chengdu, 610064, China \\ ${ }^{2}$ College of electrical engineering, Northwest University for Nationalities, Lanzhou, 730124, China \\ ${ }^{3}$ Xichang Satellite Launch Center, Xichang, 615000, China

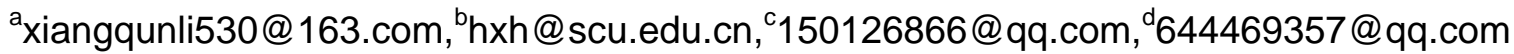

Keywords: video coding, inter-prediction, error concealment and motion compensation.

\begin{abstract}
The establishment of new generation of high efficiency video coding (HEVC) standard has been completed. There are many achievements for the research on HEVC coding efficiency, but there are few researches on HEVC based error concealment. In this paper, the packet loss of the simulation channel transmission in the HEVC test platform is designed and realized. The packet loss rate can be set at will and the packet loss location in each frame is selected randomly. Meanwhile, based on the feature of large size of HEVC coding unit, the spatio-temporal method is proposed to be used to recover lost LCU. Through experimental verification and the comparison with relevant algorithm, better repair effects are obtained by the method proposed in this paper based on the test results.
\end{abstract}

\section{Introduction}

The establishment of new generation of high efficiency video coding (HEVC) standard has been completed currently. Compared with previous H.264/AVC standard, about $50 \%$ bit rate can be reduced in HEVC standard under the condition of ensuring same video quality. Higher compression means less redundant information, leading to stronger dependence among information. Packet loss will make recreated video images blurring or lost in part, to cause great declination of play quality of decoded video, even the possibility of no real-time play. Therefore, the robustness in video transmission must be considered when the data rate obtained from HEVC coding is transmitted in complex environment or unstable network. Error concealment technique is able to effectively improve the reliability of information transmission [1], at the same time, it is an effective technology to solve the error signal at decoder [2]. It will test decoded images after each frame of images is decoded. If error information included in images is found, the error concealment system shall be started to be called to conceal the error information. Based on different repair modes, error concealment is divided into intra-frame error concealment and inter-frame error concealment, corresponding to spatial error concealment and temporal error concealment techniques, respectively.

In this paper, from the point of practical application, for the packet loss encountered in the simulation channel transmission process, the data rate packet loss module is designed and completed in the HEVC frame, to reconstruct the packet loss processing step of the simulation channel. To realize the packet loss processing of data rate at any ratio and to facilitate the normal conduction of packet loss processing and subsequent error concealment study, the output format of data rate is set as AnnexB format in this paper, simultaneously, each LCU is set as a piece during the HEVC coding process due to the consideration of two output formats of HEVC data rate. In the simulation packet loss processing of AnnexB data rate, Picture Parameter Set (PPS) and Sequence Parameter Sets (SPS) information cannot be lost. This information is related to the normal decoding process of the whole video and the data started with the prefix "00000001" should not be lost, either. Once it is lost, the normal coding and decoding of the current frame will be affected. Through the analysis above, random overall rejection can be taken for the data with the format combination of prefix "00000001" +NALU in this paper, to achieve the effects of packet loss of simulation channel. The data rate after packet loss is able to meet the demands of study on error concealment. 


\section{Proposed method}

In this paper, every lost LCU coding block is divided into two areas to be repaired respectively. Every different area is repaired by different error concealment method. As shown in Fig 1, different error concealment algorithms are selected to recover the data of the two areas in the figure, respectively.

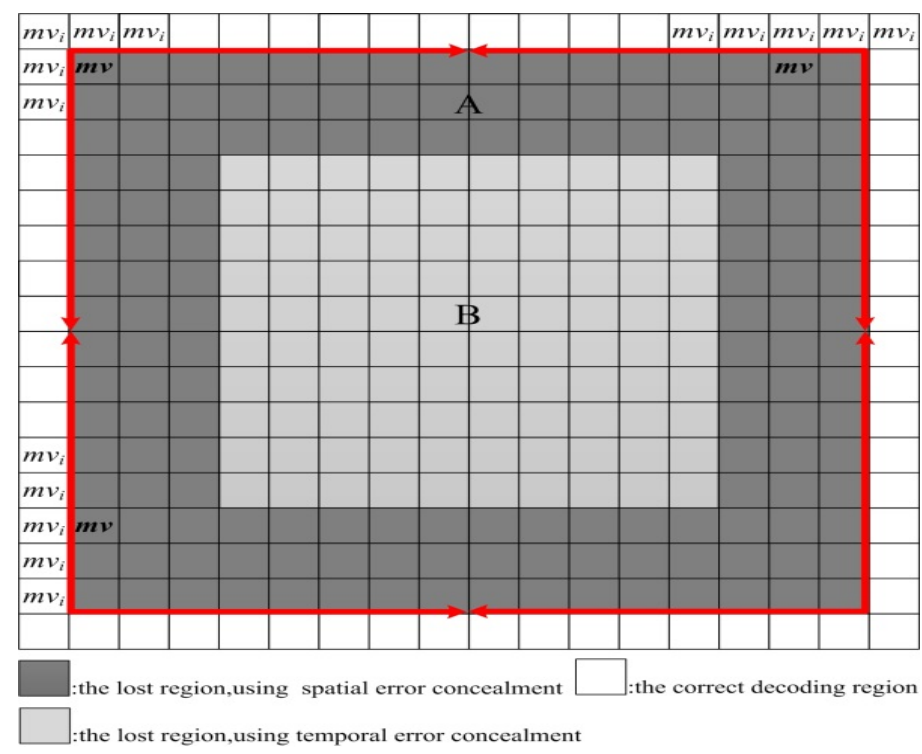

Fig 1.Temporal-spatial Error Concealment Scheme

As shown in Fig.1,Area ' $A$ ' in the figure refers to the $4^{\mathrm{X}} 4$ subblock of the peripheral three layers of the lost LCU block, close to adjacent blocks and possessing good spatial correlation with the adjacent domain block with correct periphery decoding. Spatial error concealment algorithm can be taken. Area ' $\mathrm{B}$ ' is the area excluding the peripheral three layers of the lost LCU block. Since the space distance from $4^{\times} 4$ subblocks in this area to adjacent blocks of current block is increased, causing the reduction of spatial correlation. The specific steps for the area ' $A$ ' are as follows: Step 1: take the motion vector (mvi) of five subblocks closest to the lost block. Step 2: calculate the average of mvi in the five subblocks in Step 1, and assign the average to the lost block as mv of the lost block. Step 3: find corresponding compensation data in the reference frame to recover the lost pixel through the motion vector obtained in Step 2. In the calculation process of spatial correlation-based motion vector, the mv information of the correctly decoded subblock in adjacent area of the lost subblock shall be used. The recovery sequence is shown in the arrow of Fig.1. For the lost subblocks in Area B, they are recovered by temporal correlation between adjacent frames in this paper. In Fig 2, if there are two frames or more reference frames in the sequence of reference frames, the motion vector MV1 and MV2 at the location corresponding to current lost blocks found in the reference frame fn- 1 and fn-2 are represented with $(\mathrm{x} 1, \mathrm{y} 1)$ and $(\mathrm{x} 2, \mathrm{y} 2)$. The motion vector MV0 of the lost block is represented with (x0, y0) and the distance between $\mathrm{fn}$ and $\mathrm{fn}-1, \mathrm{fn}-2$ is $\mathrm{d} 1, \mathrm{~d} 2$. So the equation below is obtained:

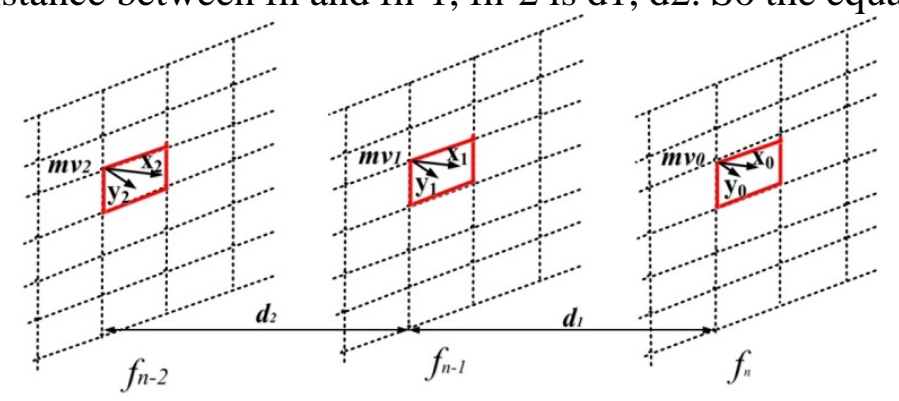

Fig 2.Variation of Motion Vector in Multiple Reference Frames

$\left(x_{0}-X_{1}\right) / d_{1}=\left(x_{1}-x_{2}\right) / d_{2}$

$\left(y_{0}-y_{1}\right) / d_{1}=\left(y_{1}-y_{2}\right) / d_{2}$

From the equation: 
$\left.x_{0}=\left(d_{1} \times\left(x_{1}-x_{2}\right)\right)\right) / d_{2}+x_{1}$

$y_{0}=\left(d_{1} \times\left(y_{1}-y_{2}\right)\right) / d_{2}+y_{1}$

Since $f_{n}, f_{n-1}$ and $f_{n-2}$ are a consecutive video sequence, with equal space among them, namely, $d_{1}=d_{2}$, therefore:

$x_{0}=2 x_{1}-x_{2}$

$y_{0}=2 y_{1}-y_{2}$

So, $\left(2 x_{1}-x_{2}, 2 y_{1}-y_{2}\right)$ is the estimated motion vector MV0 of current lost block. If the reference frames are less than two frames, MV1 is directly used to replace MV0. When adjacent blocks are lost or in inexistence, the motion vector information of adjacent blocks is unavailable. At this time, the motion vector estimation related to time domain is used as the lost motion vector. After the motion vector of each $4 \times 4$ subblock is obtained, the motion compensation is used to recover the lost

\section{The experimental results and analysis}

Seen from Table 1, compared with traditional BMA in HM standard test platform (HM10.0), the objective video quality can be improved for about $1.03 \mathrm{~dB}$ and the video quality is obviously improved through making use of the algorithm in this chapter to recover and process the video with different packet loss rates.

Table 1. The experiment result of the proposed method and comparison with BMA

\begin{tabular}{cccccc}
\hline \multirow{2}{*}{ Sequences } & Loss rate (\%) & \multicolumn{4}{c}{ PSNR(dB) } \\
\cline { 3 - 5 } & & HM10.0 & After loss & BMA & Proposed \\
\hline \multirow{2}{*}{ Keiba } & 2 & 39.58 & 17.53 & 23.28 & 24.34 \\
$832 \times 480$ & 4 & 39.58 & 14.52 & 22.33 & 22.72 \\
& 8 & 39.58 & 12.87 & 19.13 & 19.98 \\
\hline \multirow{2}{*}{ Jonny } & 2 & 42.59 & 18.32 & 37.55 & 38.71 \\
$1280 \times 720$ & 4 & 42.59 & 15.14 & 33.10 & 35.14 \\
& 8 & 42.59 & 13.21 & 29.85 & 32.82 \\
\hline \multirow{2}{*}{ Cactus } & 2 & 39.77 & 17.21 & 29.54 & 29.92 \\
$1920 \times 1080$ & 4 & 39.77 & 13.53 & 26.87 & 27.52 \\
& 8 & 39.77 & 11.33 & 24.76 & 25.45 \\
\hline \multirow{2}{*}{ Train } & 2 & 42.32 & 20.21 & 31.27 & 31.89 \\
\hline \multirow{2}{*}{ PSNR average } & 4 & 42.32 & 17.26 & 27.12 & 27.85 \\
\hline & 4 & 42.32 & 14.95 & 25.04 & 25.90 \\
\hline
\end{tabular}

\section{Conclusion}

Based on the characteristic of large coding blocks in HEVC, a spatio-temporal error concealment method is proposed in this paper. Through setting different packet loss rates for the test standard sequence with different resolutions, the subjective and objective quality of the video sequence after packet loss is obviously improved after the error concealment algorithm in this paper is used for recovery, at the same time, the performance is also improved compared with the BMA widely applied. 


\section{Acknowledgement}

This work was supported by the National Natural Science Foundations of China (No. 61471248), the Innovation project on the education for graduate students of Sichuan province (2014-Edu.-034).

\section{References}

[1]. Wang Y, Wenger S, et al. Error resilient video coding techniques [J].Signal Processing Magazine, IEEE, 2000, 17(4):61-82.

[2]. Wang Y K,Hannuksela M, et al. The error concealment feature in the H.26L test model[C].Image Processing.2002. Proceedings, 2002 International Conference on, IEEE, 2002, 2(2): II-729-II-732.

[3]. Huang S, Kuo S. Optimization of hybridized error concealment for H. 264 [J].IEEE Trans on Broadcasting, 2008, 54(3):499-516.

[4]. Zhu Feng, Zhang Wei-ming,et al. Adaptive error resilient coding based on FMO in wireless video transmission[C]Proc of the 3rd International Conference on Multimedia Information Networking and Security,2011:609-612.

[5]. Wu Zhe, Gao Shaoshuai. An adaptive multi-level matching interpolation algorithm for spatial error concealment[C]Proc of IET International Conference on Information and Communications Technologies.2013:539-543. 